\title{
SARS-CoV-2: Informações e impactos
}

\author{
SARS-CoV-2: Information and impacts \\ SARS-CoV-2: Información e impactos
}

Recebido: 03/06/2021 | Revisado: 14/06/2021 | Aceito: 15/06/2021 | Publicado: 30/06/2021

\author{
Gardênia Taveira Santos \\ ORCID: https://orcid.org/0000-0001-9357-6508 \\ Universidade Estadual do Maranhão, Brasil \\ E-mail: gardenia.santos.taveira.79025@gmail.com \\ Joabe Lima Araújo \\ ORCID: https://orcid.org/0000-0002-4806-9192 \\ Universidade de Brasília, Brasil \\ E-mail: joabearaujobiotec@gmail.com \\ Lucas Aires de Sousa \\ ORCID: https://orcid.org/0000-0002-1325-7759 \\ Universidade Federal Maranhão, Brasil \\ E-mail: lukarelio.aires@gmail.com \\ Ionara Nayana Gomes Passos \\ ORCID: https://orcid.org/0000-0003-4729-4977 \\ Universidade Federal do Maranhão, Brasil \\ E-mail: ionara.passos@gmail.com \\ Julia Augusto Vieira \\ ORCID: https://orcid.org/0000-0003-0566-9603 \\ Universidade de Brasília, Brasil \\ E-mail: julia.augusto.vieira@gmail.com \\ Hugo Vitor Menezes Cruz \\ ORCID: https://orcid.org/0000-0003-1607-1949 \\ Secretaria Municipal de Saúde de Fortuna, Brasil \\ E-mail: hugomenezes1996@gmail.com \\ Lindalva de Moura Rocha \\ ORCID: https://orcid.org/0000-0002-5544-8078 \\ Universidade Estadual do Maranhão, Brasil \\ E-mail: lindalva.nutri.ufpi@gmail.com
}

\begin{abstract}
Resumo
Em Wuhan, China, no final do ano de 2019 ocorreu um surto de pneumonia viral de causa desconhecida, até que em 7 de Janeiro de 2020 foi identificado o agente etiológico desta doença pelo Centro Chinês de Controle e Prevenção de Doenças (CDC), em que se tratava de uma Síndrome Respiratória Aguda Grave por Coronavírus 2 (SARS-CoV-2) conhecida como coronavírus 19 (COVID-19). Esse vírus já infectou até o dia 01 de junho de 2021 cerca de 170.814.464 pessoas, levando 3.551 .884 a óbito, além de gerar uma série de problemas sociais e econômicos no mundo. Assim, este estudo teve como objetivo documentar informações referentes ao SARS-CoV-2 contidas na literatura cientifica, bem como, os impactos gerados na sociedade pelo novo coronavírus. As buscas foram realizadas nas bases de dados: PubMed/MEDLINE, SciELO e BVS utilizando filtro de buscas de artigos a partir de Dezembro de 2019 a Maio de 2021, que são os meses que compreendem de estudos referente ao SARS-CoV-2. As buscas bibliográficas foram realizadas no mês de Maio de 2021 utilizando os descritores "COVID-19 and Impacts", "COVID-19 and Information", "Complication and COVID-19" e "COVID-19 and Fake News". Foram levados em considerações apenas achados no idioma inglês (americano e britânico). Dessa forma, esperamos que este estudo reforce o conhecimento dos leitores com informações sobre a COVID-19 e seus impactos na sociedade, também se almeja mensurar e identificar o vasto material cientifico que já temos disponível na literatura.
\end{abstract}

Palavras-chave: SARS-CoV-2; COVID-19; Novo coronavírus 2019; Impacto social.

\section{Abstract}

In Wuhan, China, at the end of 2019 there was an outbreak of viral pneumonia of unknown cause, until on January 7 , 2020, the etiologic agent of this disease was identified by the Chinese Center for Disease Control and Prevention (CDC) in that it was a Severe Acute Respiratory Syndrome Coronavirus 2 (SARS-CoV-2) known as coronavirus 19 (COVID-19). Until June 1, 2021, this virus has infected around 170,814,464 people, causing 3,551,884 people to die, in addition to generating a series of social and economic problems in the world. Thus, this study aimed to document information regarding SARS-CoV-2 contained in the scientific literature, as well as the impacts generated on society by the new coronavirus. Searches were performed in the following databases: PubMed/MEDLINE, SciELO, and BVS using a search filter for articles from December 2019 to May 2021, which are the months that comprise studies related 
to SARS-CoV-2. Bibliographic searches were performed in May 2021 using the descriptors "COVID-19 and Impacts", "COVID-19 and Information", "Complication and COVID-19", and "COVID-19 and Fake News". Only findings in the English language (American and British) were taken into consideration. Thus, we hope that this study reinforces readers' knowledge with information about COVID-19 and its impacts on society. It also aims to measure and identify the vast scientific material that we already have available in the literature.

Keywords: SARS-CoV-2; COVID-19; Novel coronavirus 2019; Social impact.

\section{Resumen}

En Wuhan, China, a finales de 2019 se produjo un brote de neumonía viral de causa desconocida, hasta que el 7 de enero de 2020 el agente etiológico de esta enfermedad fue identificado por el Centro Chino para el Control y la Prevención de Enfermedades (CDC) en el sentido de que fue un coronavirus 2 del síndrome respiratorio agudo severo (SARS-CoV-2) conocido como coronavirus 19 (COVID-19). Hasta el 1 de junio de 2021, este virus ha infectado a alrededor de 170.814 .464 personas, provocando la muerte de 3.551 .884 personas, además de generar una serie de problemas sociales y económicos en el mundo. Así, este estudio tuvo como objetivo documentar la información sobre el SARS-CoV-2 contenida en la literatura científica, así como los impactos generados en la sociedad por el nuevo coronavirus. Las búsquedas se realizaron en las siguientes bases de datos: PubMed/MEDLINE, SciELO y BVS mediante un filtro de búsqueda de artículos desde Diciembre de 2019 hasta Mayo de 2021, que son los meses que comprenden los estudios relacionados con el SARS-CoV-2. Las búsquedas bibliográficas se realizaron en Mayo de 2021 utilizando los descriptores "COVID-19 and Impacts", "COVID-19 and Information", "Complication and COVID-19" y "COVID-19 and Fake News". Solo se tomaron en consideración los hallazgos en el idioma inglés (americano y británico). Así, esperamos que este estudio refuerce el conocimiento de los lectores con información sobre COVID-19 y sus impactos en la sociedad, además de medir e identificar el vasto material científico que ya tenemos disponible en la literatura.

Palabras clave: SARS-CoV-2; COVID-19; Nuevo coronavirus 2019; Impacto social.

\section{Introdução}

Em dezembro de 2019 houve uma série de casos de uma doença respiratória aguda atípica em Wuhan, China. Essa doença se disseminou rapidamente causando um caos no sistema de saúde da cidade de Wuhan, onde só em janeiro de 2020 foi descoberto o agente etiológico responsável pelo surto, que se tratava de uma nova Síndrome Respiratória Aguda Grave por Coronavírus 2 (SARS-CoV-2 ou 2019-nCoV) (Yuki et al., 2020). Este novo patógeno possui semelhanças homologas com a Síndrome Respiratória Aguda Grave por Coronavírus (SARS-CoV) que acometeu toda a China nos anos de 2002 a 2003 , levando a morte de milhares de chineses (Ksiazek et al., 2020).

O surto do 2019-nCoV iniciou-se através de uma transmissão zoonótica, em um mercado de frutos do mar localizado em Wuhan. Em seguida já era observada a transmissão local de humano para humano, que contribuiu na disseminação da doença por toda a China e no mundo ( $\mathrm{Li}$ et al., 2020). O novo coronavírus possui RNA de fita simples com sentido positivo e faz parte dá família CoV (Lu et al., 2020) este é o sétimo CoV identificado que infecta humanos e a terceira cepa que pode levar graves complicações respiratórias, sendo as outras duas a Síndrome Respiratória do Oriente Médio (MERS-CoV) e o SARS-CoV (Mackenzie \& Smith, 2020).

As pessoas infectadas por SARS-CoV-2 apresentam uma série de sintomas, sendo que $80 \%$ apresentam sintomas leves e moderados ou nenhum sintoma (assintomáticos), 15\% avançam para uma pneumonia grave e 5\% apresentam Síndrome do Desconforto Respiratório Agudo (SDRA), choque séptico e falência de órgãos o que pode levar a morte (Huang et al., 2020). Segundo o Centro de Ciência e Engenharia de Sistemas (CSSE) da Universidade de John Hopkins, até o dia 01 de Junho de 2021, cerca de 170.814.464 casos de COVID-19 foram confirmados no mundo e uma taxa de 3.551 .884 mortes (JHU, 2021).

Atualmente existem dois tratamentos alternativos no enfrentamento da SARS-CoV-2, o Remdesivir e um coquetel que contém a combinação de casirivimabe e imdevimabe (Regn-CoV2), aprovados pela Agencia de Vigilância Sanitária (ANVISA) do Brasil (Garcia \& Pinheiro, 2021). Associado a isso, diversos fármacos e terapias estão sendo testadas para evidenciar sua eficácia em ensaios pré-clínicos e clínicos, mas nenhum destes ainda se mostrou efetivos no tratamento da COVID-19 (Pacheco et al., 2020). Dessa forma, a imunização com vacinas na quarta fase de estudos e o distanciamento social 
são as formas mais efetivas de conter a rápida propagação do vírus, sendo recomendado pela Organização Mundial da Saúde (OMS) e adotado por diversos países (Yuki et al., 2020).

Deste modo, este estudo apresentará uma revisão narrativa da literatura científica de artigos publicados sobre o SARSCoV-2, buscando informações que são de utilidade no meio social e acadêmico, pois essa crise sanitária é um problema de saúde pública em todo o mundo, acometendo mais de 200 países, sendo atualmente os Estados Unidos da América (EUA), Índia e o Brasil os países mais afetados com 33.269.749, 28.175.044 e 16.545.544 casos confirmados, respectivamente. Levando países ao colapso no sistema de saúde, além de problemas econômicos, sociais e sanitários (Madabhavi et al., 2020; JHU, 2021).

\section{Metodologia}

Trata-se de um estudo de revisão narrativa, conforme o protocolo usado em estudos de Torres et al. (2021), com algumas modificações. As bases de dados usadas para as pesquisas bibliográficas foram: National Center for Biotechnology Information (PubMed/MEDLINE), Scientific Electronic Library Online (SciELO) e Biblioteca Virtual em Saúde (BVS). Essas são bases de dados que concentram publicações de excelência voltadas na área da saúde. As buscas dos artigos publicados nas bases de dados foi compreendida no período de Maio de 2021 e para a seleção dos estudos foi utilizado filtro de buscas no período de Dezembro de 2019 a Maio de 2021, pois são os meses que compreendem de estudos referente ao SARS-CoV-2. Os descritores utilizados foram: "COVID-19 and Impacts", "COVID-19 and Information", "Complication and COVID-19" e "COVID-19 and Fake News". Foram levados em considerações apenas artigos publicados no idioma inglês (americano e britânico).

\section{Desenvolvimento}

\section{SARS-CoV-2}

No dia 31 de dezembro do ano de 2019, foi informado e notificado ao Escritório da OMS situado na China, sobre alguns casos novos de pneumonia atípica em Wuhan, essa pneumonia despertou a atenção não só da China, mas de diversos países no mundo (Shi et al., 2020). Com isso, as autoridades da China iniciaram investigações para definir que tipo de doença estava enfrentando e conhecê-la. Dessa forma, criaram protocolos sanitários e aplicaram medidas de isolamento social para diminuir a propagação do vírus e também criaram procedimentos para diagnósticos e possíveis tratamentos, no entanto as medidas não foram suficientes para evitar uma disseminação mundial causando uma pandemia (Wang et al., 2020a).

\section{Aspectos gerais e etiológicos}

O vírus da COVID-19 teve sua primeira identificação em um mercado de frutos do mar de Wuhan, China. Neste local, animais silvestres eram vendidos de forma ilegal (Nowicki et al., 2020). Com isso, a OMS por meio do Comitê Internacional de Taxonomia de Vírus classificou esta doença como SARS-CoV-2 ou 2019-nCoV (Szcześniak et al., 2020). Esse vírus pertence à subfamília Coronavirinae, da ordem Nidovirales. Essa família de coronavirus se divide em quatro gêneros $\alpha$ coronavírus, $\beta$-coronavírus, $\gamma$-coronavírus e $\Delta$-coronavírus. Os $\alpha$-coronavírus e $\beta$-coronavírus infectam apenas mamíferos. Já os $\gamma$-coronavírus e 4-coronavírus infectam aves, no entanto alguns desses podem se adaptar ao novo microambiente, acometendo assim mamíferos (Woo et al., 2012).

O vírus da COVID-19 possui um genoma com RNA de cadeia única, sendo positiva, com uma organização característica de outros coronavírus (Zhu et al., 2020). Algumas análises do genoma da SARS-CoV-2 mostraram que o novo coronavírus compartilham semelhanças estruturais e biológicas com outras espécies da família CoV. Além de apresentar uma estrutura similar, grande parte dos CoVs partilham um meio de infecção semelhante (Hulswit et al., 2016). Existem evidencias 
de que o neurotropismo é uma dessas semelhanças que os CoVs tem em comum (Szcześniak et al., 2020). E devido a grande prevalência e extensa distribuição dos coronavírus, juntamente com uma diversidade genética, recombinação constante, e ação entre humanos e animais, pode ocorrer o surgimento de novos coronavírus em eventos de spillover ocasionais (Cui et al., 2019).

\section{Epidemiologia da SARS-CoV-2 no mundo}

A cronologia das infecções pela SARS-CoV-2 se inicia com o primeiro caso notificado em dezembro de 2019 em Wuhan, China (Du Toit, 2020). Com isso, do dia 18 a 29 de Dezembro de 2019 cinco pacientes foram hospitalizados com SDRA, dos cinco pacientes um acabou indo a óbito (Ren et al., 2020). No dia 22 de Janeiro de 2020 foram confirmados 571 novos casos da nova doença COVID-19 em 25 províncias da China (Lu, 2020). Em seguida, no dia 30 de Janeiro de 2020 a situação já se alastrava pelo mundo, 7.734 casos foram notificados na China, e 90 casos notificados em outros países, são eles: Taiwan, Tailândia, Vietnã, Malásia, Nepal, Sri Lanka, Camboja, Japão, Cingapura, República da Coreia, Emirados Árabes Unidos, EUA, Filipinas, Índia, Austrália, Canadá, Finlândia, França e Alemanha, em que a letalidade estava em uma taxa de 2,2\% (Bassetti et al., 2020). Atualmente, após elevada disseminação da SARS-CoV-2 em centenas de países, até o dia 04 de Maio de 2021 já havia 153.676.825 casos confirmados em todo o mundo e 3.216.016 mortes. Tendo como os três países mais afetados pela pandemia os EUA com 32.477.036 casos, Índia com 20.282.833 casos, e o Brasil com 14.779.529 casos (JHU, 2021).

\section{Epidemiologia da SARS-CoV-2 no Brasil}

Percebendo que a situação do novo coronavírus estava crescendo de forma descontrolada em números de casos e afetando centenas de países, a OMS declarou no dia 30 de Janeiro de 2020 Emergência de Saúde Pública de Importância Internacional (ESPII). Já no Brasil, em 3 de fevereiro do mesmo ano foi anunciado Emergência de Saúde Pública de Importância Nacional (ESPIN) (Garcia \& Duarte, 2020). A pandemia foi declarada pela OMS em 11 de Março de 2020, e nesse dia já havia mais de 110 mil casos e 4 mil mortes em todos os continentes do mundo (WHO, 2020).

A Lei $n^{\circ} 13.979$ foi sancionada no Brasil pelo excelentíssimo Senhor Presidente da República com a finalidade de dispor métodos para o enfrentamento da COVID-19 no país (BRASIL, 2020). As Unidades da Federação (UFs) começaram a executar as medidas na Lei ${ }^{\circ} 13.979$ na segunda semana de março de 2020. Em especial os estados federativos do Rio de Janeiro, São Paulo, Santa Catarina e o Distrito Federal, que nos dias que se seguiram ao dia 24 de Março agiram com medidas mais rigorosas de quarentena (Garcia \& Duarte, 2020).

Até o dia 9 de Maio de 2020, o Brasil já havia sido notificado cerca de 147 mil casos e 10 mil óbitos ocasionados pela SARS-CoV-2, e juntamente com isso a transmissão comunitária foi oficialmente reconhecida e declarada em todo o país (Dong et al., 2020). As estratégias adotadas pelo Ministério da Saúde (MS) do Brasil, em discordância com o chefe do poder executivo para diminuir a propagação do novo coronavírus ainda estão sendo o distanciamento social e medidas de isolamento nos casos de contaminação, visando evitar um colapso da assistência hospitalar devido a crescente demanda de leitos de internação (Daumas et al., 2020).

\section{Fatores de risco}

Alguns estudos mostraram que comorbidades existentes em pacientes infectados pela SARS-CoV-2 podem desencadear um prognóstico ruim (Wang et al., 2020b). Associado a isso, resultados de uma pesquisa de revisão sistemática com meta-análise evidenciaram que indivíduos do sexo masculino, com idade superior a 65 anos e fumantes podem ter uma grande possibilidade de progredirem para a condição crítica e, as comorbidades presentes como hipertensão, diabetes, doenças 
cardiovasculares ou respiratórias podem prejudicar de forma significativa o prognóstico da COVID-19 (Zheng et al., 2020). Devido a grande alta das taxas de mortalidade e acelerada propagação da SARS-CoV-2, é totalmente necessário à avaliação dos possíveis fatores de risco que podem agravar a progressão da doença em indivíduos diagnosticados como o novo coronavírus (Wang et al, 2020c; Yu et al., 2020).

\section{Prevenção e tratamento}

O período de incubação da SARS-CoV-2 ocorre por volta de 2 a 14 dias (Singhal, 2020). Muitos dos casos da COVID-19 são propagados de pessoa para pessoa antes do aparecimento dos sintomas, isso acontece em $44 \%$ dos casos, demonstrando dificuldade na contenção da disseminação do vírus, como também a relevância de medidas preventivas como a higiene das mãos, distanciamento social e uso de máscaras (Pacheco et al., 2020). É recomendado o isolamento domiciliar de pessoas diagnosticadas com a COVID-19 ou com sintomas suspeitos da doença. A ventilação nos locais, em especial nas residências como casas e apartamentos deve ser constante e com luz solar. Os pacientes internados em hospitais ou UTIs devem usar máscara cirúrgica simples e fazer a higiene pessoal constantemente. Os responsáveis pelos cuidados dos doentes devem também usar máscara cirúrgica e higienizar as mãos a cada 15-20 minutos (Singhal, 2020).

No que se refere ao tratamento, atualmente existem dois tratamentos alternativos no enfrentamento da SARS-CoV-2 no Brasil, o remdesivir e o coquetel Regn-CoV2, ambos aprovados pela ANVISA (Garcia \& Pinheiro, 2021) e as vacinas Janssen e Coronavac (uso emergencial) e AstraZeneca e Pfizer (registro definitivo) também aprovadas pela ANVISA como uso na quarta fase dos testes clínicos com proposta de vacinação em massa (Leme, 2021). Países que compõe a União Europeia (Alemanha, Grécia, Áustria, Hungria, Bélgica, Irlanda, Bulgária, Itália, Chéquia, Letónia, Chipre, Lituânia, Croácia, Luxemburgo, Dinamarca, Malta, Eslováquia, Países Baixos, Eslovénia, Polónia, Espanha, Portugal, Estónia, Roménia, Finlândia, Suécia e França) também aprovaram o uso emergencial do remdesivir e o esteroide dexametasona para o tratamento alternativo da COVID-19, Assim como os EUA (Deutsch et al., 2020) e o Canadá que aprovou o uso do remdesivir em 27 de Julho de 2020 pela sua agência governamental canadense (Canada, 2021), além das vacinas desenvolvidas (Le et al., 2020).

Além disso, diversos medicamentos já existentes no mercado farmacêutico e utilizados no tratamento de outras doenças estão sendo avaliados para o redirecionamento no tratamento da COVID-19. São eles: baricitinibe, atazanavir, o anticorpo monoclonal do receptor da interleucina-6 (IL-6) tocilizumabe e os medicamentos anti-influenza favipiravir e umifenovir (Araújo et al., 2021; Triggle et al., 2020).

\section{Considerações Finais}

Os estudos mostram que a pandemia ainda não foi controlada em países com dificuldades na aquisição de vacinas, como é o caso do Brasil. Em que esses países dependem de medidas restritivas como o distanciamento social, uso de máscaras e isolamento de pessoas diagnosticadas com a SARS-CoV-2. No entanto, métodos de tratamentos alternativos estão sendo usados e investigados para ajudar a controlar a doença e evitar possíveis colapsos em seus sistemas de saúde, como o Brasil, Canadá, EUA e todos os 27 países que compõem a União Europeia utilizam o remdesivir. Ressaltamos imprescindivelmente que o método efetivo para controlar a pandemia da COVID-19 é a vacina e que métodos alternativos de tratamento estão sendo utilizados para tentar evitar um colapso no sistema de saúde por dificuldades de aquisição das mesmas (vacinas).

\section{Agradecimentos}

Os autores agradecem a colaboração do Grupo de Pesquisa em Química Medicinal e Biotecnologia - QUIMEBIO da Universidade Federal do Maranhão - UFMA, Campus Universitário de São Bernardo e ao Grupo de Pesquisa em Ciências 
Naturais e Biotecnologia - CIENATEC da Universidade Federal do Maranhão - UFMA, Campus Universitário de Grajaú e a Coordenação de Aperfeiçoamento de Pessoal de Nível Superior - CAPES pela bolsa de estudo.

\section{Referências}

Araújo, J. L., Sousa, L. A. D., Sousa, A. O., Bastos, R. S., Santos, G. T., Lage, M. R., \& Rocha, J. A. (2021). DFT, molecular docking, and ADME/Tox screening investigations of market-available drugs against SARS-CoV-2. Journal of the Brazilian Chemical Society, 00(00), 1-14. https://dx.doi.org/10.21577/0103-5053.20210061

Bassetti, M., Vena, A., \& Giacobbe, D. R. (2020). The novel Chinese coronavirus (2019-nCoV) infections: Challenges for fighting the storm. European Journal of Clinical Investigation, 50(3), e13209. https://doi.org/10.1111/eci.13209

Brasil. LEI No 13.979, de 6 de fevereiro de 2020. Secretaria-Geral Subchefia para Assuntos Jurídicos. 2020. http://www.planalto.gov.br/ccivil_03/_ato20192022/2020/lei/L13979.htm/.

Canada. (2021). Drugs and vaccines for COVID-19. Canada.ca. https://www.canada.ca/en/health-canada/services/drugs-health-products/covid19industry/drugs-vaccines-treatments/about.html.

Cui, J., Li, F., \& Shi, Z. L. (2019). Origin and evolution of pathogenic coronaviruses. Nature Reviews Microbiology, 17(3), 181-192. https://doi.org/10.1038/s41579-018-0118-9

Daumas, R. P., Silva, G. A., Tasca, R., Leite, I. D. C., Brasil, P., Greco, D. B., ... \& Campos, G. W. D. S. (2020). O papel da atenção primária na rede de atenção à saúde no Brasil: limites e possibilidades no enfrentamento da COVID-19. Cadernos de Saúde Pública, 36, e00104120. https://doi.org/10.1590/0102311X00104120

Deutsch, A., Ptak, A., Guarascio, F. (2020). Países europeus enfrentam escassez de remdesivir para tratar covid-19. AgênciaBrasil. https://agenciabrasil.ebc.com.br/internacional/noticia/2020-10/paises-europeus-enfrentam-escassez-de-remdesivir-para-tratar-covid-19.

Dong, E., Du, H., \& Gardner, L. (2020). An interactive web-based dashboard to track COVID-19 in real time. The Lancet infectious diseases, 20(5), 533-534. https://doi.org/10.1016/S1473-3099(20)30120-1

Du Toit, A. (2020). Outbreak of a novel coronavirus. Nature Reviews Microbiology, 18(3), 123-123. https://doi.org/10.1038/s41579-020-0332-0

Garcia, L. P., \& Duarte, E. (2020). Intervenções não farmacológicas para o enfrentamento à epidemia da COVID-19 no Brasil. Epidemiologia e Serviços de Saúde, 29(2), e2020222. https://doi.org/10.5123/S1679-49742020000200009

Garcia, M.; Pinheiro, L. (2021). Anvisa autoriza uso emergencial de coquetel de anticorpos contra Covid-19. https://g1.globo.com/bemestar/coronavirus/noticia/2021/04/20/anvisa-autoriza-uso-emergencial-de-coquetel-contra-covid-19.ghtml.

Huang, C., Wang, Y., Li, X., Ren, L., Zhao, J., Hu, Y., \& Cao, B. (2020). Clinical features of patients infected with 2019 novel coronavirus in Wuhan, China. The lancet, 395(10223), 497-506. https://doi.org/10.1016/S0140-6736(20)30183-5

Hulswit, R. J. G., De Haan, C. A. M., \& Bosch, B. J. (2016). Coronavirus spike protein and tropism changes. Advances in virus research, 96, $29-57$. https://doi.org/10.1016/bs.aivir.2016.08.004

JHU - Johns Hopkins University. 2021. Coronavirus COVID-19 Global Cases by the Center for Systems Science and Engineering (CSSE). Johns Hopkins University, 2021. https://gisanddata.maps.arcgis.com/apps/opsdashboard/index.html\#/bda7594740fd40299423467b48e9ecf6/.

Ksiazek, T. G., Erdman, D., Goldsmith, C. S., Zaki, S. R., Peret, T., Emery, S., \& SARS Working Group. (2003). A novel coronavirus associated with severe acute respiratory syndrome. New England journal of medicine, 348(20), 1953-1966. https://doi.org/10.1056/NEJMoa030781

Le, T. T., Andreadakis, Z., Kumar, A., Román, R. G., Tollefsen, S., Saville, M., \& Mayhew, S. (2020). The COVID-19 vaccine development landscape. Nature Reviews Drug Discovery, 19(5), 305-306. https://doi.org/10.1038/d41573-020-00073-5

Leme, L. (2021). Veja quais são as vacinas contra covid-19 já aprovadas para aplicação no Brasil. https://www.acidadeon.com/campinas/cotidiano/va cinas/NOT,0,0,1600052,confira-as-vacinas-contra-covid-19-ja-aprovadas-para-aplicacao-no-brasil.aspx.

Li, Q., Guan, X., Wu, P., Wang, X., Zhou, L., Tong, Y., \& Feng, Z. (2020). Early transmission dynamics in Wuhan, China, of novel coronavirus-infected pneumonia. New England journal of medicine, 382, 1199-1207. https://doi.org/10.1056/NEJMoa2001316

Lu, H. (2020). Drug treatment options for the 2019-new coronavirus (2019-nCoV). Bioscience trends, 14(1), 69-71. https://doi.org/10.5582/bst.2020.01020

Lu, R., Zhao, X., Li, J., Niu, P., Yang, B., Wu, H., ... \& Tan, W. (2020). Genomic characterisation and epidemiology of 2019 novel coronavirus: implications for virus origins and receptor binding. The lancet, 395(10224), 565-574. https://doi.org/10.1016/S0140-6736(20)30251-8

Mackenzie, J. S., \& Smith, D. W. (2020). COVID-19: a novel zoonotic disease caused by a coronavirus from China: what we know and what we don't. Microbiology Australia, 41(1), 45-50. https://doi.org/10.1071/MA20013

Madabhavi, I., Sarkar, M., \& Kadakol, N. (2020). COVID-19: a review. Monaldi Archives for Chest Disease, 90(2), 248-258. https://doi.org/10.4081/monaldi.2020.1298

Nowicki, G. J., Ślusarska, B., Tucholska, K., Naylor, K., Chrzan-Rodak, A., \& Niedorys, B. (2020). The severity of traumatic stress associated with COVID19 pandemic, perception of support, sense of security, and sense of meaning in life among nurses: Research protocol and preliminary results from Poland. International journal of environmental research and public health, 17(18), 6491. https://doi.org/10.3390/ijerph17186491 
Pacheco, T. J. A., Souza, D. G. D., Lima, L. I. D., \& Longo, J. P. F. (2020). Panorama mundial de estudos com a hidroxicloroquina para o tratamento da COVID-19. Journal of Health \& Biological Sciences, 8(1), 1-4. http://dx.doi.org/10.12662/2317-3076jhbs.v8i1.3288.p1-4.2020

Ren, L. L., Wang, Y. M., Wu, Z. Q., Xiang, Z. C., Guo, L., Xu, T., \& Wang, J. W. (2020). Identification of a novel coronavirus causing severe pneumonia in human: a descriptive study. Chinese medical journal, 133(9), 1015-1024. http://dx.doi.org/10.1097/CM9.0000000000000722

Shi, Y., Wang, J., Yang, Y., Wang, Z., Wang, G., Hashimoto, K., \& Liu, H. (2020). Knowledge and attitudes of medical staff in Chinese psychiatric hospitals regarding COVID-19. Brain, Behavior, \& Immunity-Health, 4, 100064. https://doi.org/10.1016/j.bbih.2020.100064

Singhal, T. (2020). A review of coronavirus disease-2019 (COVID-19). The indian journal of pediatrics, 87(4), 281-286. https://doi.org/10.1007/s12098-020$03263-6$

Szcześniak, D., Gładka, A., Misiak, B., Cyran, A., \& Rymaszewska, J. (2020). The SARS-CoV-2 and mental health: From biological mechanisms to social consequences. Progress in Neuro-Psychopharmacology and Biological Psychiatry, 104(10), e110046. https://doi.org/10.1016/j.pnpbp.2020.110046

Torres, J. I. da S. L., Araújo, J. L., Vieira, J. A., Souza, C. dos S., Passos, I. N. G., Rocha, L. de M. (2021). Endometriosis, difficulties in early diagnosis and female infertility: A review. Research, Society and Development, 10(6), e6010615661. http://dx.doi.org/10.33448/rsd-v10i6.15661

Triggle, C. R., Bansal, D., Abd Farag, E. A. B., Ding, H., \& Sultan, A. A. (2020). COVID-19: learning from lessons to guide treatment and prevention interventions. mSphere Journal, 5(3), e00317-20. https://doi.org/10.1128/mSphere.00317-20

Wang, B., Li, R., Lu, Z., \& Huang, Y. (2020b). Does comorbidity increase the risk of patients with COVID-19: evidence from meta-analysis. Aging (Albany NY), 12(7), 6049-6057. https://dx.doi.org/10.18632\%2Faging.103000

Wang, C., Horby, P. W., Hayden, F. G., \& Gao, G. F. (2020a). A novel coronavirus outbreak of global health concern. The lancet, 395(10223), 470-473. https://doi.org/10.1016/S0140-6736(20)30185-9

Wang, D., Hu, B., Hu, C., Zhu, F., Liu, X., Zhang, J., \& Peng, Z. (2020c). Clinical characteristics of 138 hospitalized patients with 2019 novel coronavirusinfected pneumonia in Wuhan, China. Jama, 323(11), 1061-1069. http://jamanetwork.com/article.aspx?doi=10.1001/jama.2020.1585

WHO - World Health Organization. 2020. Rolling updates on coronavirus disease (covid-19). Genebra: World Health Organization. Disponível em: https://www.who.int/emergencies/diseases/novel-coronavirus-2019/events-as-they-happen/. Acesso em: 04 Jan. 2021.

Woo, P. C., Lau, S. K., Lam, C. S., Lau, C. C., Tsang, A. K., Lau, J. H., \& Yuen, K. Y. (2012). Discovery of seven novel Mammalian and avian coronaviruses in the genus deltacoronavirus supports bat coronaviruses as the gene source of alphacoronavirus and betacoronavirus and avian coronaviruses as the gene source of gammacoronavirus and deltacoronavirus. Journal of virology, 86(7), 3995-4008. https://dx.doi.org/10.1128\%2FJVI.06540-11

Yu, F., Du, L., Ojcius, D. M., Pan, C., \& Jiang, S. (2020). Measures for diagnosing and treating infections by a novel coronavirus responsible for a pneumonia outbreak originating in Wuhan, China. Microbes and infection, 22(2), 74-79. https://doi.org/10.1016/j.micinf.2020.01.003

Yuki, K., Fujiogi, M., \& Koutsogiannaki, S. (2020). COVID-19 pathophysiology: A review. Clinical immunology, 108427. https://doi.org/10.1016/j.clim.2020.108427

Zheng, Z., Peng, F., Xu, B., Zhao, J., Liu, H., Peng, J., \& Tang, W. (2020). Risk factors of critical \& mortal COVID-19 cases: A systematic literature review and meta-analysis. Journal of Infection, 81(2), e16-e25. https://doi.org/10.1016/j.jinf.2020.04.021

Zhu, N., Zhang, D., Wang, W., Li, X., Yang, B., Song, J., \& Tan, W. (2020). A novel coronavirus from patients with pneumonia in China, 2019. New England journal of medicine, 382, 727-733. https://doi.org/10.1056/NEJMoa2001017 